\title{
Emotional Adjustment in Couples During and After a Failed IVF Procedure: A Systematic Review
}

\author{
Dr. Rishi Mishra ${ }^{1 *}$, Mr. Ashwani Mohan ${ }^{2}$
}

\section{ABSTRACT}

This review aims to access the emotional setbacks and changes faced by couple who start with the IVF treatment and face a negative outcome after the treatment. It systematically analyses the level of anxiety faced by the couple before, during and after the IVF treatment cycles. Studies show that unsuccessful treatment raised the couple's anxiety level and the levels of negative emotions, which showed significant presence even after consecutive unsuccessful cycles. Further findings indicated that unsuccessful IVF couples reported more emotional distress relative to those whom treatment had worked and females in particular experienced more psychosocial stress as compared with males. Couples who had at least one child prior to IVF treatment experienced greater emotional distress at follow-up. These couples felt less supported by the IVF Unit staff and were less satisfied with the counseling they received. The results highlighted the importance of post-treatment counseling when treatment is unsuccessful.

Keywords: Anxiety/Depression/Emotional Response/Fertility Treatment/IVF/ Adjustment

IVF (in vitro fertilization) is the most common form of ART (Assisted Reproductive Technology) for pregnancy issues. If the fallopian tubes are not properly functioning or the sperm is of poor quality, it turns out to be the only treatment left. Since its inception it has proved to be a remarkable pregnancy treatment even in women with hopelessly damaged fallopian tubes, seemingly sterile husbands, and even "unsolved” infertility.

According to The Society for Assisted Reproductive Technology (SART) the chances in young and fertile couple is just a $15-20 \%$ to conceive naturally in any one .Women with top chances of IVF success have per-cycle success rates of $40 \%$ or higher, while the majority of women have per-cycle success rates of 20-35\%.[1,2] In reality, the IVF success is affected by many factors in

\footnotetext{
${ }^{1}$ Visiting Faculty, Amity Institute of Psychology and Allied Sciences, Amity University, Noida , U.P, India.

${ }^{2}$ Assistant Professor, Amity Institute of Psychology and Allied Sciences, Amity University, Noida , U.P, India *Responding Author

(C) 2016, R Mishra, A Mohan; licensee IJIP. This is an Open Access Research distributed under the terms of the Creative Commons Attribution License (http://creativecommons.org/licenses/by/2.0), which permits unrestricted use, distribution, and reproduction in any Medium, provided the original work is properly cited.
} 
addition to age, such as your height, weight, ovarian reserve tests, sperm count, reproductive history, and clinical diagnoses.[3]

Because of these issues the emotional adjustment to IVF has received ample attention in clinical, descriptive and empirical studies [4]. These studies have primarily addressed the taxing consequences of infertility and its management. Using representative samples and standardized measures, empirical research conducted in the 1980s, however, demonstrated that, in general, infertile couple does not differ significantly from control or norm groups on emotional aspects [5]. This review demanded that longitudinal and prospective studies were needed to sustain these results and to uncover information about groups at risk of emotional troubles as a result of unsuccessful fertility treatment.

Recent years have seen a substantial expansion of research efforts into couple's emotional adjustment to IVF. A systematic and comprehensive overview of over the last 25 years specifically targets the process and the multidimensional character of emotional adjustment to unsuccessful treatment [5].

\section{METHODOLOGY}

This review comprises a number of researches and studies published in peer reviewed journals, the electronic databases of Medline, PubMed and PsychInfo. Articles pertaining to Emotional distress related to fertility issues and IVF were examined.

\section{The level of anxiety and emotional stress faced by the couple before IVF treatment cycles}

In the review process we consider the psychological adjustment mechanism in relation to the diverse characteristics and stages of the medical course of action by differentiating between the different cycles of an IVF treatment. The number of treatment options left could easily determine the emotional response to the treatment and its outcome. For the same reason, this review exclusively focuses on IVF as it is nearly always the last treatment option for couples with fertility problems. Accordingly, we do not consider studies on emotional adjustment to less invasive treatment options such as intrauterine insemination.

IVF is a multidimensional stressor; the treatment itself constitutes the primary stressor and is most likely to evoke anxiety. The unpredictable outcome of the treatment is another major stress inducing agent, more likely to evoke feelings of depression [6].

Following stress-coping models on adjustment to a chronic stressor, our review regards the emotional response to IVF in terms of anxiety, depression and general distress. Aspects of coping skills, social support and personality characteristics are considered determinants of the emotional response and are thus taken into account as risk and protective factors to explain 


\section{Emotional Adjustment in Couples During and After a Failed IVF Procedure: A Systematic Review}

individual differences in emotional response to unsuccessful IVF based on stress-coping models $[6,7,8]$

A sound insight into such risk and protective factors would facilitate the identification of couple at risk of experiencing emotional problems and foster the development of preventative and tailored support.

\section{Emotional response to IVF Pretreatment emotional adjustment}

Nine studies investigated emotional adjustment before the start of fertility treatment. Six studies provided information about pretreatment depression levels using different inventories: three used the Beck Depression Inventory (BDI) (Beck et al., 1961), one the Symptom Checklist (SCL-90) (Derogatis et al., 1973), one the Centre for Epidemiologic Studies Depression Scale (CES-D) (Radloff, 1977) and two the Profile of Mood States (POMS) (McNair et al., 1971).

Result of Studies: None of the studies reported differences in depression between the IVF patients and norm groups. All studies also assessed anxiety, most frequently the STAI (Spielberger, 1983) or SCL-90. With respect to state anxiety, four studies did not report differences with norm groups [9], whereas five showed enhanced pretreatment levels of state anxiety.[10]

Pretreatment anxiety scores on the STAI differed considerably. This is partly explained by the differences in norms, may be because of cultural aspects and the type of norm group. As anxiety scores differ per age and sex, normative data should match these variables. However, most studies failed to provide information about the characteristics of the norm groups.

Still, the differences in norm groups could not explain the considerable differences in pretreatment state anxiety levels even within one country. [11] The time in which the study was conducted (i.e. just after the introduction of IVF or more recently) could not explain the disparity in the state anxiety findings nor were there differences related to type of sample.

In addition, the variations were also present in studies assessing state anxiety at more or less the same moment: immediately before the start of the medication. Nor could age differences explain the variations: the mean age of the couple participating in the studies varied from 32 to 34 years. It is known that ways of communicating fertility problems and treatment possibilities, just as the method of delivering information, tend to influence the patients' emotional responses before the start of IVF.

The differences in pretreatment emotional adjustment may hence have been attributable to the different patient approaches the studies adhered to, but, unfortunately, the studies presented did not provide any details on these aspects of their procedures.

(c) The International Journal of Indian Psychology, ISSN 2348-5396 (e) | ISSN: 2349-3429 (p) | 7 


\section{Emotional Adjustment in Couples During and After a Failed IVF Procedure: A Systematic Review}

All but one study assessed anxiety with general inventories that were unrelated to the fertility problem. Of special interest is the study by Fekkes et al. (2003) that took both general and fertility related emotional problems into account. It found no deviations from the norm groups on the general instruments but did report higher levels of emotional complaints for the fertility related measures (Fekkes et al., 2003).

In summary, we can say that before the start of the treatment, in general, IVF patients did not disagree from norm groups with respect to depression levels, whereas the results on state anxiety were ambiguous: some studies showed elevated levels in the patients, other studies found no difference compared to norm groups.

\section{The level of anxiety faced by the couple in between the IVF treatment cycles}

In this five studies assessed couple's anxiety and general distress during the course of one treatment cycle[12,13].The study of Boivin and Takefman (1995) warrants special mention because they evaluated the entire course of the IVF treatment cycle by having the couple keep a daily record of subjective general distress, starting at day 1 of the cycle and ending after the result of the pregnancy test had been obtained.

Distress levels did not show remarkable changes in the first segment of the treatment cycle, despite a slight increase at OPU. A more significant increase of distress was reported at the end of the cycle, just before the pregnancy test (Boivin and Takefman, 1995). Other studies, too, indicated a similar increase in distress during the course of the cycle. Overall, OPU and pregnancy test proved to constitute the most stressful stages of the IVF cycle [12,13].

\section{Emotional adjustment after the IVF treatment cycles}

Six studies repeated their assessments of couple's emotional response before and after one or more treatment cycles.Three are of special interest because they discussed the results of couple after both successful and unsuccessful treatments [14] Hynes et al., 1992; Lok et al., 2002) investigated the emotional response to unsuccessful treatment only.

The most consistent finding reported by three studies was an increase in depression after one or more unsuccessful treatment cycles showing significant interaction effects between time and treatment outcome [14].

Results: The results with respect to anxiety after unsuccessful treatment were less consistent: of the three studies that assessed anxiety, two showed an increase in anxiety levels relative to baseline after unsuccessful treatment [16]: time $\square$ outcome: $F=6.5$; $P<0.01$; Newton et al., 1990: time: $F=25.2 ; P<0.01$ ). 


\section{Emotional Adjustment in Couples During and After a Failed IVF Procedure: A Systematic Review}

Visser et al. (1994) did not find any pre-post-treatment differences. However, the results of the latter study are difficult to interpret because the researchers differentiated between subgroups based on the number of treatment cycles before pregnancy.

Only one study evaluated the course of anxiety and depression several months after treatment conclusion and reported no recovery from enhanced anxiety and depression levels in this period after unsuccessful treatment [15,16].

The results with respect to the emotional adjustment to successful treatment consistently showed equally low or decreased pre-to-post-treatment anxiety and depression levels, reflecting that negative emotions regarding IVF disappear after successful treatment.

In general, unsuccessful treatment tends to evoke an increase of at least depressive symptoms, which continue for a longer period of time after treatment.

The pre-post results are based on averages of the emotional adjustment observed in couple after successful and unsuccessful treatment Studies investigating emotional response during one treatment cycle DACL, Depression Adjective Checklist (Lubin, 1981); MAACL, Mean Affect Adjective Checklist (Zuckerman and Lubin, 1965); PANAS, Positive and Negative. Affect Scale (Watson et al., 1988); POMS, Profile of Mood States (McNair et al., 1971); STAI, Spielberger State in Trait Anxiety Inventory (Spielberger, 1983).

Verhaak et al. (2001). Couple who may have clinical levels of emotional problems constitute a group that may also need additional psychological support during fertility treatment. Three studies reported proportions varying from 10 (Lok et al., 2002) to 25\% (Verhaak et al., 2005b; Newton et al., 1990) of couple with clinically relevant levels of depression after one or more unsuccessful treatment cycles.

Although most of the couple therefore seem to adjust well to unsuccessful treatment, a considerable number of patients might be eligible for supplemental counseling.

\section{Long-term post-treatment emotional adjustment}

The six studies we found on long-term emotional adjustment to IVF that met the criteria for review there were no longitudinal studies on the emotional response of couple after IVF treatment had been abandoned. There were two cross-sectional studies that compared the emotional adjustment of couple after unsuccessful treatment with that of couple after successful IVF [15].

Remarkably, Hammarberg et al. (2001) did not report any differences in emotional status between these two contrasting groups several years after treatment cessation. In contrast, the 


\section{Emotional Adjustment in Couples During and After a Failed IVF Procedure: A Systematic Review}

earlier study by Freeman et al. (1987) did find higher levels of depression in the couple who had not conceived following IVF compared with the controls (couple with children).

The discrepancy in findings may be because of the variations in the time elapsed since the final treatment cycle.

In sum, longitudinal research into couple's emotional response after unsuccessful IVF is lacking, and cross-sectional studies showed couple after unsuccessful fertility treatment to have a less positive affect than couple with successful IVF but are inconsistent about the group's differences in negative affect.

\section{Predictors of adjustment to infertility}

Compared with studies in the emotional adjustment to IVF, studies in the prediction of the emotional response to unsuccessful treatment are relatively scarce. This does not tally with the relevance of this type of research for the psychological support for IVF patients as it can provide essential information about protective and risk factors for adaptation problems facilitating the identification of couple at risk at an early stage. Few studies with solid prospective designs showed that cognitive processes play an important role in the adjustment to unsuccessful treatment.

\section{Recommendations for future research}

This review provides several starting points for future research. First, there is a clear need for further longitudinal studies that follow couple in the last phase of their IVF treatment through 23 years after abandoning treatment to shed more light on the final adaptation process, i.e. a cognitive shift from trying to get pregnant to trying to give meaning to one's life without a child that is genetically one's own.

Furthermore, future studies into the emotional response within one treatment cycle should differentiate between the stress of the treatment itself and the stress caused by the threat of infertility.

\section{Clinical implications}

Negative emotional responses proved to be strongly related to the outcome of the treatment, i.e. to the threat of definitive childlessness. Accordingly, psychological support should be specifically targeted to help the woman adjust to the possibility of treatment failure and eventual childlessness rather than to help her to cope with the impact of the treatment itself.

As potential studies showed, especially the pretreatment cognitions of helplessness and acceptance with respect to possible childlessness are the factors that play a prominent role in determining the emotional response to treatment failure. From the start, additional psychosocial 


\section{Emotional Adjustment in Couples During and After a Failed IVF Procedure: A Systematic Review}

care should be dedicated to change the meaning of childlessness. Fertility professionals can advance the process of acceptance by discussing the infertility problems with the couples and by improving their communications about the issue, i.e. by asking about possible plans in the event of unsuccessful treatment and by gauging possible differences in motivation for treatment between the spouses (Boivin et al., 2001; Kentenich et al., 2002). Clinicians should also prepare their patients for possible emotional reactions to unsuccessful treatment. Indeed, Hammarberg et al. (2001) reported that the couples themselves indicated a need for information on the emotional aspects of their fertility problems. Such psychosocial education, e.g. explaining to the couple in advance that enhanced distress is a natural reaction to unsuccessful treatment, might enhance their control over their emotional response to treatment failure. In most cases, the knowledge will reassure the couple that what they are experiencing is part of a normal reaction and not an indication of dysfunctional adjustment.

Again, because everlasting infertility and concluding childlessness constitute the most important stressor in IVF treatment, any psychological intervention should be aimed at the couple's acceptance of their fertility problems and adjustment to the probability of childlessness.

To summarize, most couple adjust well to unsuccessful IVF cycles, but there is a lack of awareness about the process of adjustment to definitive unsuccessful treatment and childlessness as well as about the factors that contribute to the process. Beyond the considerable negative emotional response, most couple experience after abandoning treatment, which represents a normal grief reaction that is expected to diminish over time, there is a sizeable number of couple who develops or are at risk of developing severe emotional problems after IVF cessation.

It is the need of the hour to pay more attention to those couple for whom the treatment does not lead to offspring and to find better support strategies for these couples.

\section{Acknowledgments}

The author appreciates all those who participated in the study and helped to facilitate the research process.

\section{Conflict of Interests}

The author declared no conflict of interests.

\section{REFERENCES}

1. Assisted reproductive technology in the United States: 2001 results generated from the American Society for Reproductive Medicine/Society for Assisted Reproductive Technology registry. Fertility and sterility. Jun 2007;87(6):1253-1266.

2. Sunderam S, Chang J, Flowers L, et al. Assisted reproductive technology surveillance-United States, 2006. MMWR Surveill Summ. Jun 12 2009;58(5):1-25. 


\section{Emotional Adjustment in Couples During and After a Failed IVF Procedure: A Systematic Review}

3. Banerjee P, Choi B, Shahine LK, et al. Deep phenotyping to predict live birth outcomes in in vitro fertilization. Proc Natl Acad Sci U S A. Aug 3 2010;107(31):13570-13575.

4. Menning, 1980; Greenfield et al., 1984; Freeman et al.1985; Mahlstedt, 1985; Hearn et al., 1987; Callan and Hennessey, 1988; Dennerstein and Morse, 1988; Kemeter, 1988.

5. $\quad$ Dunkel-Schetter and Lobel, 1991Dunkel- Schetter and Lobel, 1991.

6. Abramson et al., 1978; Beck et al., 1979; Eysenck, 1981.

7. Lazarus and Folkman, 1984; Leventhal et al.,1984; Cohen and Wills, 1985; Holahan and Moos, 1985

8. $\quad$ Ormel and Wohlfart, 1991; Edelmann, 1992; Clark et al., 1994; Costa et al., 1996; Holahan et al., 1996; Alloy et al., 1999.

9. Hearn et al., 1987; Edelmann et al., 1994; Verhaak et al., 2001; Fekkes et al., 2003

10. Beaurepaire et al., 1994; Visser et al., 1994; Mori et al., 1997; Slade et al., 1997; Salvatore et al., 2001

11. Visser et al., 1994; Verhaak et al., 2001

12. Merari et al., 1992; Boivin and Takefman, 1995; Ardenti et al., 1999; Yong et al.

13. Kolonoff-Cohen et al., 2001

14. Visser et al., 1994:

15. Slade et al., 1997.

16. Verhaak et al. 2001, 2005.

How to cite this article: R Mishra, A Mohan (2016), Emotional Adjustment in Couples During and After a Failed IVF Procedure: A Systematic Review, International Journal of Indian Psychology, Volume 4, Issue 1, No. 69, ISSN:2348-5396 (e), ISSN:2349-3429 (p), DIP:18.01.002/20160401, ISBN:978-1-365-45447-9 